\title{
Searches for long-lived particle decays in ATLAS
}

\section{Daniela Salvatore ${ }^{* \dagger}$ on behalf of the ATLAS Collaboration}

E-mail: daniela.salvatoredcern.ch

\begin{abstract}
Searches for the decay of neutral, weakly interacting, long-lived particles using $20.3 \mathrm{fb}^{-1}$ of 2012 data collected at $8 \mathrm{TeV}$ by the ATLAS detector at the LHC are presented. The first analysis is sensitive to long-lived particles that decay to Standard Model particles producing jets at the outer edge of the ATLAS electromagnetic calorimeter or inside the hadronic calorimeter. The second search employs techniques for reconstructing decay vertices of long-lived particles decaying to jets in the inner detector and muon spectrometer. No significant excess of events over the expected background are found, and limits as a function of proper lifetime are reported for the decay of the Higgs boson and other scalar bosons to long-lived particles and for Z' and Stealth SUSY benchmark models.
\end{abstract}

The European Physical Society Conference on High Energy Physics

22-29 July 2015

Vienna, Austria

* Speaker.

${ }^{\dagger}$ INFN Cosenza, Italy. 


\section{Physics motivation}

Many extensions to the Standard Model (SM) include the production of particles that are neutral, weakly-coupled and long-lived that can decay to final-states containing several hadronic jets, as gauge-mediated Supersymmetry Breaking (GSMB), the Minimal Supersymmetric Standard Model (MSSM) with R-parity violation, inelastic dark matter, Stealth Supersymmetry (SUSY) ([1], [2]), Hidden Valley (HV) scenarios ([3], [4]), and Two Higgs Doublet Models (2HDM). In particular, three models are studied with the ATLAS experiment ([5]):

- $\Phi$ as a communicator (Fig. 1a): a SM-sector scalar boson $\Phi$ (that may not necessarily be the SM Higgs boson $(\mathrm{H}))$ mixes with $\Phi_{\mathrm{hs}}$, a hidden sector scalar boson which can decay to v-quarks $\left(q_{\mathrm{v}}\right)$. The hidden sector consists of a confining gauge group that makes v-hadrons out of its v-quarks, in analogy with QCD. The v-quarks then hadronize to v-particles that can decay back to SM particles. The lightest HV particles $\pi_{\mathrm{v}}$ are pair-produced and each decays to a pair of SM fermions. The current Higgs boson measurement uncertainties do not exclude non-SM decay modes at the $30 \%$ or more level, so the decay of the SM $125 \mathrm{GeV}$ Higgs boson to a pair of long-lived scalars or pseudo scalars is also explored. The lifetime of the $\pi_{\mathrm{v}}$ is unconstrained and could be quite long. If a $\pi_{\mathrm{v}}$ decays in the inner detector (ID) or in the muon spectrometer (MS), it can be reconstructed as a non-standard displaced vertex (DV). A $\pi_{\mathrm{v}}$ that instead decays deep inside the calorimeter is reconstructed as a jet with an unusual energy signature that most traditional searches reject as having poor data quality.

- Z' as a communicator (Fig. 1b): a massive communicator, Z', produced by quark-antiquark annihilation decays into the hidden sector via $Z^{\prime} \rightarrow q_{\mathrm{v}} \bar{q}_{\mathrm{v}}$. The v-quarks hadronize into showers of $\pi_{\mathrm{v}}$ particles. The $\pi_{\mathrm{v}}^{ \pm}$lifetime is a free parameter.

- Stealth SUSY (Fig. 2): a class of R-parity-conserving SUSY models that do not have large missing transverse momentum $\left(E_{\mathrm{T}}^{\text {miss }}\right)$ signatures. This model involves adding a hiddensector (stealth) singlet superfield $\mathrm{S}$ at the electroweak scale, which has a superpartner singlino $\tilde{S}$. The SUSY decay chain ends with $\tilde{S}$ decaying to a singlet plus a low-mass gravitino $(\tilde{G})$, where $\tilde{G}$ carries off very little energy and the singlet promptly decays to two gluons. The effective decay processes are $\tilde{g} \rightarrow \tilde{S} g$ (prompt), $\tilde{S} \rightarrow S \tilde{G}$ (not prompt), and $S \rightarrow g g$ (prompt). This scenario results in one prompt gluon and two displaced gluon jets per gluino decay resulting in two DVs.

\section{Decays in the Hadronic Calorimeter}

A detailed description of the analysis strategy, which looks for long-lived particle (LLP) decays in the hadronic calorimeter (HCal) from the HV process depicted in Fig. 1a, is available in [6].

The list of simulated datasets is reported in Table 1: on the left side, the $\Phi$ mass or Higgs boson mass, the $\Phi$ gluon fusion production cross section, and $\pi_{\mathrm{v}}$ mass of each benchmark model generated are reported. On the right side, the decay branching ratios (BR) of the $\pi_{\mathrm{v}}$ as a function of the $\pi_{\mathrm{v}}$ mass are listed.

Based on the characteristic signatures of these samples, the following analysis cuts have been optimised: 
- Trigger selection: the dedicated CalRatio trigger ([7]) looks specifically for LLPs that decay near the outer radius of the electro-magnetic calorimeter (ECal) or within the HCal: events containing at least one narrow jet with little energy deposited in the ECal are selected. Jets must satisfy $E_{\mathrm{T}}>35 \mathrm{GeV}$ and $\log _{10}\left(E_{\mathrm{H}} / E_{\mathrm{EM}}\right)>1.2$, where $\left(E_{\mathrm{H}} / E_{\mathrm{EM}}\right)$ is the ratio of the energy in the $\mathrm{HCal}\left(E_{\mathrm{H}}\right)$ to the energy in the $\mathrm{ECal}\left(E_{\mathrm{EM}}\right)$. Also they must have no tracks with transverse momentum $p_{\mathrm{T}}>1 \mathrm{GeV}$ in the region $0.2 \times 0.2(\Delta \eta \times \Delta \phi)$ around their axes.

- Event selection: a standard good primary vertex is reconstructed in the event. Events are rejected if at least one reconstructed jet shows evidence of being caused by a beam-halo interaction. In addition, a $E_{\mathrm{T}}^{\text {miss }}<50 \mathrm{GeV}$ cut is applied to reject cosmic rays or beam-halo interactions.

- Offline jet selection: jets are reconstructed, from the calorimeter energy clusters, with the anti- $k_{\mathrm{T}}$ algorithm ([8]) with $\mathrm{R}=0.4$; at least one jet must fire the trigger and have $E_{\mathrm{T}}>60$ $\mathrm{GeV}$ and a second jet must have $E_{\mathrm{T}}>40 \mathrm{GeV}$. Both jets must satisfy the same constraints of the trigger jet on $\log _{10}\left(E_{\mathrm{H}} / E_{\mathrm{EM}}\right)$ and ID isolation, which are very powerful handles to reject QCD background (Fig. 3). The energy-weighted average of the timing for each cell in the jet in the $[-1 ; 5]$ ns range is used to reject cosmic or beam-halo events.

The QCD di-jet background has been estimated through a multi-jet data sample, where events are selected by single-jet triggers with $E_{\mathrm{T}}>15 \mathrm{GeV}$, must have $E_{\mathrm{T}}^{\text {miss }}<50 \mathrm{GeV}$, at least two back-toback $(\Delta \Phi>2.0)$ jets with $E_{\mathrm{T}}>40 \mathrm{GeV}$ and $|\eta|<2.5$. The second (probe) jet is used to measure two probabilities: the probability $\mathrm{P}$ for a jet to pass the trigger and the $E_{\mathrm{T}}>60 \mathrm{GeV}$ requirement and the probability $\mathrm{Q}$ for a jet to pass the $E_{\mathrm{T}}>40 \mathrm{GeV}$ requirement. For both $\mathrm{P}$ and $\mathrm{Q}$, the jet must also pass $\log _{10}\left(E_{\mathrm{H}} / E_{\mathrm{EM}}\right)>1.2$, track isolation, and all other selection requirements. The probabilities $\mathrm{P}$ and $\mathrm{Q}$ are then combined into an event probability using combinatorics. With this technique, $23.2 \pm 8.0$ (stat. $\oplus$ syst.) events are estimated for the QCD di-jet background in the signal region.

Cosmic rays may deposit energy in the calorimeters without passing through the ID. The overall contribution is reduced by the jet-timing and $E_{\mathrm{T}}^{\text {miss }}$ cut. The CalRatio trigger version active only during an empty crossing was used. Each proton beam is divided up into buckets, most of which are filled with protons. An empty crossing occurs when an empty bucket in each beam

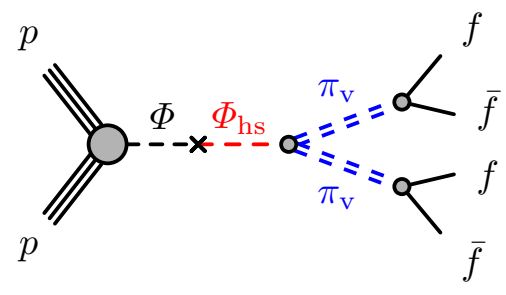

(a)

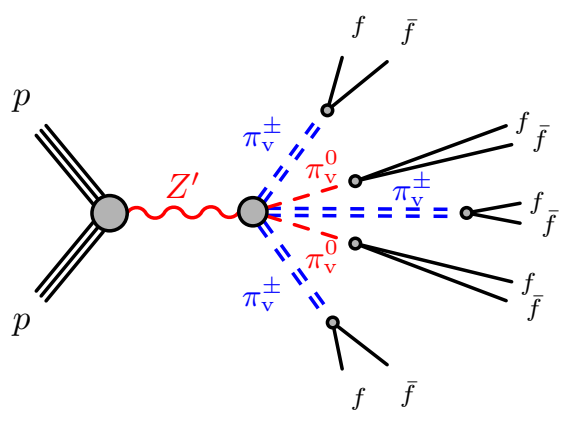

(b)

Figure 1: The HV processes: (a) $\Phi$ as a communicator, (b) Z' as a communicator. 


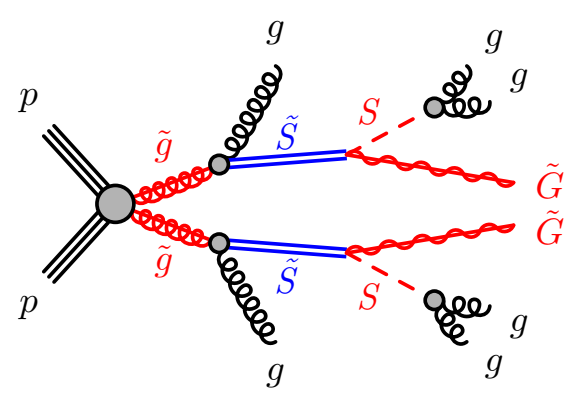

(a)

Figure 2: The Stealth SUSY process.

coincides in the centre of the detector, and five buckets on either side in each beam are also empty. The predicted number of cosmic-ray events is $0.3 \pm 0.2$.

Expected events from signal MC samples can range up to hundred of events and observed collision are fully compatible with background expectations. The CLs method ([9]) has been used to derive upper limits on the $\sigma(\Phi) \times B R\left(\Phi_{\mathrm{hs}} \rightarrow \pi_{\mathrm{v}} \pi_{\mathrm{v}}\right)$ as a function of $\mathrm{H} / \Phi$ mass, the $\pi_{\mathrm{v}}$ mass and $\pi_{\mathrm{v}}$ proper decay lengths from 0 to $50 \mathrm{~m}$ (Fig. 4 and Fig. 5).

\begin{tabular}{c|c|c}
\hline $\begin{array}{c}m_{\mathrm{H}} \\
{[\mathrm{GeV}]}\end{array}$ & $\begin{array}{c}\sigma \\
{[\mathrm{pb}]}\end{array}$ & $\begin{array}{c}\pi_{\mathrm{v}} \text { mass } \\
{[\mathrm{GeV}]}\end{array}$ \\
\hline \hline 126 & 19.0 & $10,25,40$ \\
\hline \hline$\Phi$ mass \\
{$[\mathrm{GeV}]$} & $\begin{array}{c}\sigma \\
{[\mathrm{pb}]}\end{array}$ & $\begin{array}{c}\pi_{\mathrm{v}} \text { mass } \\
{[\mathrm{GeV}]}\end{array}$ \\
\hline \hline 100 & 29.7 & 10,25 \\
140 & 15.4 & $10,20,40$ \\
300 & 3.59 & 50 \\
600 & 0.52 & 50,150 \\
900 & 0.06 & 50,150 \\
\hline \hline
\end{tabular}

\begin{tabular}{c|c|c|c}
\hline $\begin{array}{c}\pi_{\mathrm{v}} \text { mass } \\
{[\mathrm{GeV}]}\end{array}$ & $\begin{array}{c}\mathrm{BR} b \bar{b} \\
{[\%]}\end{array}$ & $\begin{array}{c}\mathrm{BR} \tau^{+} \tau^{-} \\
{[\%]}\end{array}$ & $\begin{array}{c}\mathrm{BR} c \bar{c} \\
{[\%]}\end{array}$ \\
\hline \hline 10 & 70.0 & 16.4 & 13.4 \\
20 & 86.3 & 8.0 & 5.6 \\
25 & 86.6 & 8.1 & 5.3 \\
40 & 86.5 & 8.5 & 5.0 \\
50 & 86.2 & 8.8 & 4.9 \\
150 & 84.8 & 10.2 & 4.8 \\
\hline \hline
\end{tabular}

Table 1: The $\Phi$ mass or Higgs boson mass, $\Phi$ gluon fusion production cross section, and $\pi_{\mathrm{v}}$ mass of each benchmark model generated. The cross-sections are based on the assumption that the $\Phi$ boson production mechanism is the same as the Higgs boson production mechanism. The decay branching ratios of the $\pi_{\mathrm{v}}$ as a function of the $\pi_{\mathrm{v}}$ mass are listed in the second table on the right.

\section{Decays in the Inner Detector and in the Muon Spectrometer}

A parallel search ([10]) was performed in order to look for LLP decays in the ID or the MS from the three processes in Fig. 1 and Fig. 2.

Two different trigger algorithms have been used to select different topologies:

- the dedicated Muon RoI Cluster trigger ([7]), used for scalar boson (Fig. 1a) and Stealth SUSY (Fig. 2) searches looking for 2 MS DVs or an ID DV + a MS DV, selects hadronic 


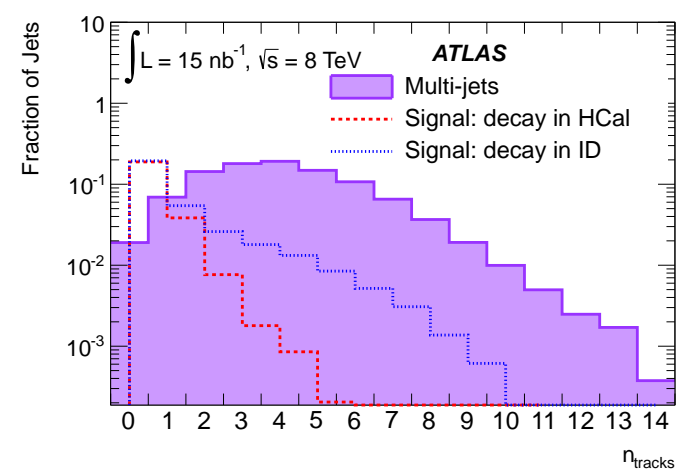

(a)

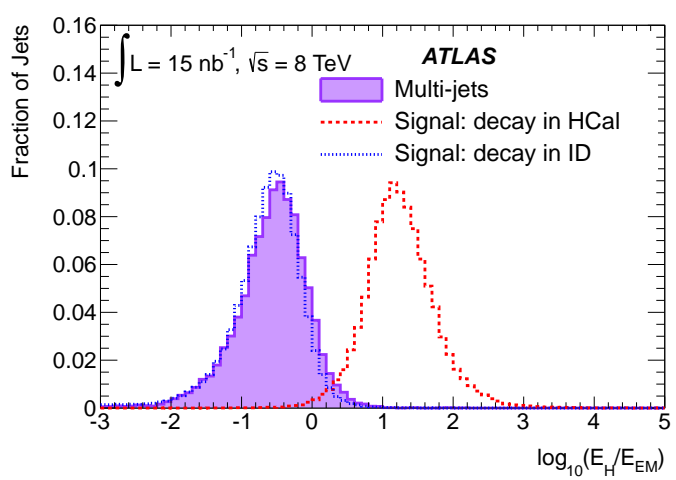

(b)

Figure 3: [6] (a) Number of good tracks ( $n_{\text {tracks }}$ ) with $p_{\mathrm{T}}>1 \mathrm{GeV}$ and $\Delta R<0.2$ around the jet axis and (b) jet $\log _{10}\left(E_{\mathrm{H}} / E_{\mathrm{EM}}\right)$ with jet $|\eta|<2.5, p_{\mathrm{T}}>40 \mathrm{GeV} . \pi_{\mathrm{v}}$ jets decaying in the HCal or in the ID are taken from the $m_{\mathrm{H}}=126 \mathrm{GeV}, m_{\pi v}=10 \mathrm{GeV}$ sample. Events are required to satisfy $E_{\mathrm{T}}^{\text {miss }}<50 \mathrm{GeV}$.

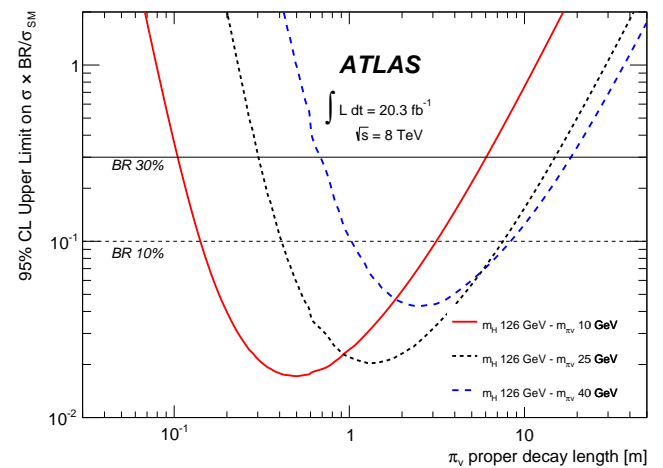

Figure 4: [6] Observed 95\% CL limits on $\sigma / \sigma_{\mathrm{SM}}$ for $m_{\mathrm{H}}=126 \mathrm{GeV}$ samples as a function of the $\pi_{\mathrm{v}}$ proper decay length. Assuming $\sigma_{\mathrm{SM}}=19.0 \mathrm{pb}$, the horizontal solid line corresponds to $\mathrm{BR}=30 \%$ and the horizontal dashed line to $\mathrm{BR}=10 \%$.

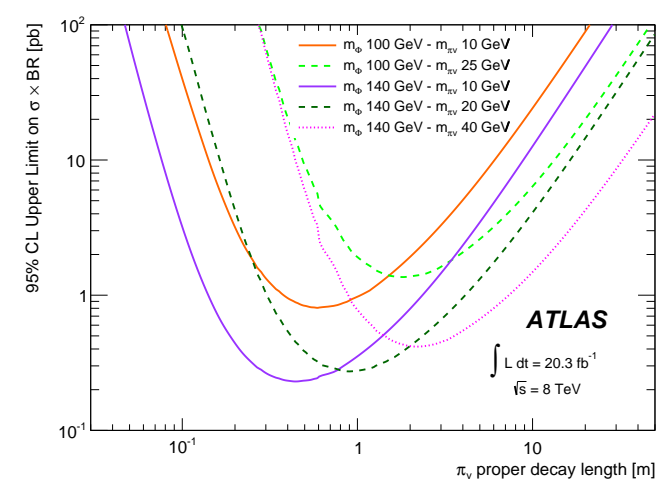

(a) Low mass samples.

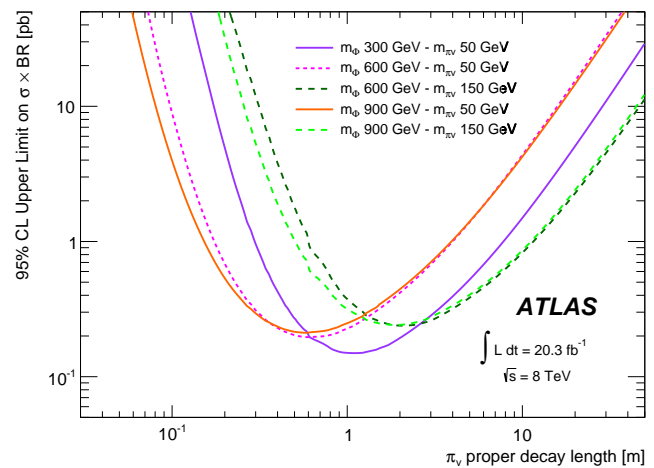

(b) High mass samples.

Figure 5: [6] (a) Observed 95\% CL limits on $\sigma \times B R[p b]$ for low and (b) high-mass $\Phi$ samples. 
decays from the outer radius of the HCal to the middle of the MS. The selected events show a cluster of muon RoIs in a $\Delta R=0.4$ cone and with little or no activity in the ID or calorimeters.

- a Jet $+E_{\mathrm{T}}^{\text {miss }}$ trigger (110 and $75 \mathrm{GeV}$ thresholds, respectively) is used for Z' searches (Fig. 1b) looking for 2 ID DVs, or 2 MS DVs or an ID DV + a MS DV.

For the scalar boson searches, boson masses from 100 to $900 \mathrm{GeV}$ and $\pi_{\mathrm{v}}$ masses from 10 to $50 \mathrm{GeV}$ are used; Z' masses of 1 or $2 \mathrm{TeV}$ are used together with $\pi_{\mathrm{v}}$ of 50 or $120 \mathrm{GeV}$; $\tilde{g}$ masses from 110 to $1200 \mathrm{GeV}$ are chosen together with $\tilde{S}$ and $\mathrm{S}$ of 100 and $90 \mathrm{GeV}$ respectively.

Displaced tracks are reconstructed by using, in a second iteration of track finding, the unassociated hits from the standard tracking algorithm ([11]). The algorithm to identify secondary vertices in the ID is based on the ATLAS primary vertex reconstruction algorithm, but with looser constraints on track impact parameters and the number of silicon, pixel and TRT hits. In simulation, reconstructed vertices must be within $5 \mathrm{~mm}$ of the simulated LLP decay position. Vertices in the MS are reconstructed by track segments from hits in the two multilayers of an MDT chamber and pairing the segments to form tracklets. Detectable decay vertices are located between the outer edge of the $\mathrm{HCal}$ and before the middle station of the muon chambers. Good vertices must be isolated with respect to ID tracks and jets and have hundreds of hits in MDT and RPC: vertex reconstruction efficiency ranges from 30 to $40 \%$ in the barrel region and up to $70 \%$ in the endcaps.

The expected number of signal events are of the order of tens for the ID DV + MS DV channel and of hundreds for the 2 MS DVs channel, while the observed events are 0 and 2 respectively. They are fully compatible with background expectations, so that CLs method was used to derive upper limits (Fig. 6 and Fig. 7) on $\sigma \times B R$ : BR for $\pi_{\mathrm{v}}$ decay to fermion pairs, as well as BRs for $\tilde{S} \rightarrow S \tilde{G}$ and $S \rightarrow g g$, are assumed to be $100 \%$. From the upper limits shown in Fig. 6, which are the most stringent up to date, the ranges of $\pi_{\mathrm{v}}$ lifetimes excluded at $30 \%, 15 \%, 5 \%$, or $1 \% \mathrm{BR}$ are extracted and reported in Table 2.

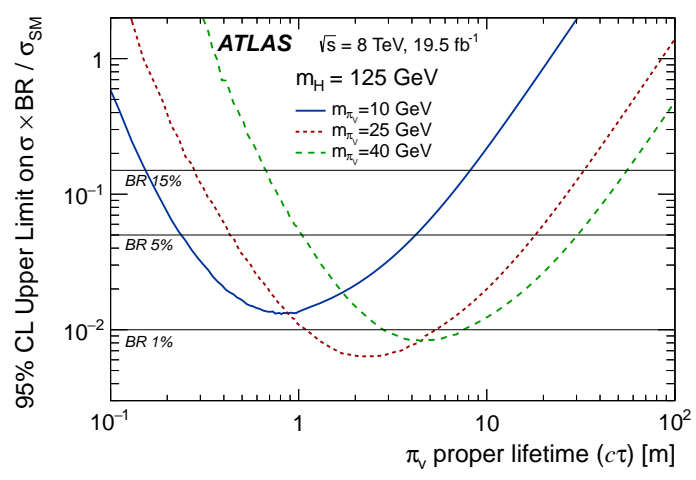

Figure 6: [10] Observed 95\% CL limits on $\sigma / \sigma_{\mathrm{SM}}$ for $m_{\mathrm{H}}=125 \mathrm{GeV}$ samples as a function of the $\pi_{\mathrm{v}}$ proper decay length. Three horizontal lines mark branching fractions for the Higgs boson decaying to $\pi_{\mathrm{v}}$ pairs at $15 \%, 5 \%$, and $1 \%$. 


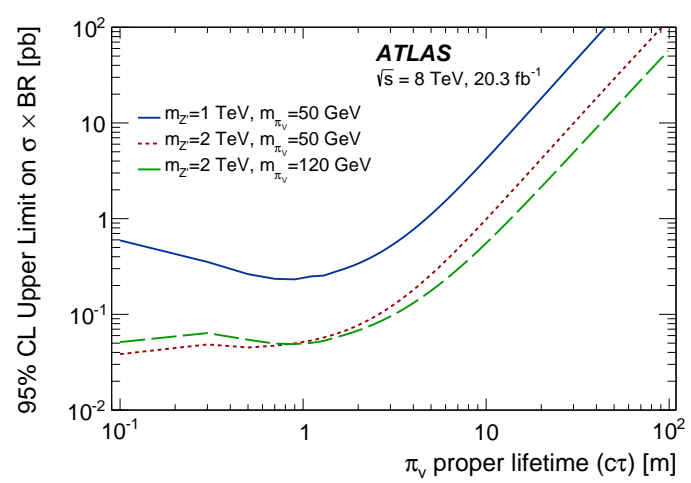

(a) Z' model.

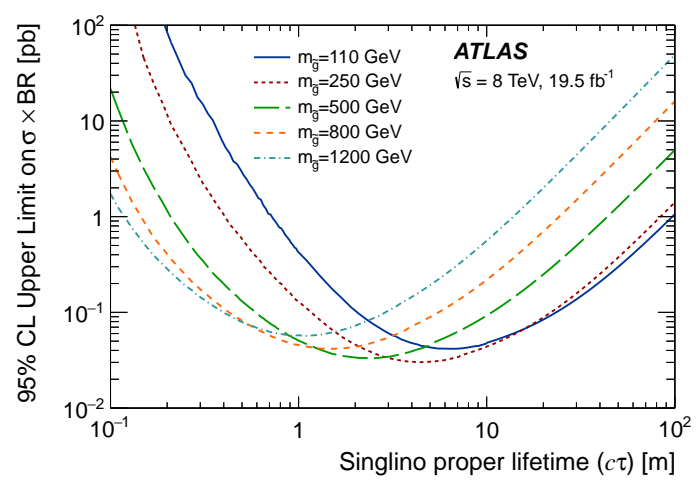

(b) Stealth SUSY model.

Figure 7: [10] Observed 95\% CL limits on $\sigma \times$ BR [pb] for (a) Z' benchmark model and (b) Stealth SUSY samples.

\begin{tabular}{|c|c|c|c|c|}
\hline \multirow{2}{*}{$\begin{array}{c}m_{\pi v} \\
{[\mathrm{GeV}]}\end{array}$} & \multicolumn{4}{|c|}{ Excluded $c \tau$ range $[\mathrm{m}]$} \\
\hline & $1 \% \mathrm{BR}$ & $5 \% \mathrm{BR}$ & $15 \% \mathrm{BR}$ & $30 \% \mathrm{BR}$ \\
\hline 10 & no limit & $0.24,4.2$ & $0.16,8.1$ & $0.12,11.8$ \\
\hline 25 & 1.10 & $0.43,18.1$ & $0.28,32.8$ & $0.22,46.7$ \\
\hline 40 & $2.82,7.45$ & $1.04,30.4$ & $0.68,55.5$ & $0.52,79.2$ \\
\hline
\end{tabular}

Table 2: Ranges of $\pi_{\mathrm{v}}$ proper decay lengths excluded at $95 \% \mathrm{CL}$ assuming a $30 \%, 15 \%$, $5 \%$, or $1 \%$ BR for $m_{H}=125 \mathrm{GeV}$.

\section{Conclusions}

Results of two searches for the decay of long-lived neutral particles to hadronic jets using pp collisions at $8 \mathrm{TeV}$ recorded at the LHC with the ATLAS detector are presented. Event selection uses three triggers: the CaloRatio trigger $\left(20.3 \mathrm{fb}^{-1}\right)$, the Muon RoI Cluster trigger $\left(19.5 \mathrm{fb}^{-1}\right)$ and a Jet $+E_{\mathrm{T}}^{\text {miss }}$ trigger $\left(20.3 \mathrm{fb}^{-1}\right)$. A final state with jets in the hadronic calorimeter plus five final states with two displaced vertices are investigated, employing techniques for reconstructing highly displaced decays in the inner detector and muon spectrometer.

No significant excess of events above the background expectations is observed and exclusion limits as a function of the proper lifetime of long-lived particles from Higgs boson and scalar boson decays are reported. For the first time, upper limits as a function of proper lifetime for Hidden Valley Z' and Stealth SUSY scenarios are shown. For the SM Higgs boson decaying to long-lived particles, significant ranges of $\pi_{\mathrm{v}}$ proper lifetimes are excluded.

\section{References}

[1] J. Fan, M. Reece, and J. T. Ruderman, Stealth Supersymmetry, JHEP 1111 (2011) 012.

[2] J. Fan, M. Reece, and J. T. Ruderman, A Stealth Supersymmetry Sampler, JHEP 1207 (2012) 196.

[3] M. J. Strassler and K. M. Zurek, Phys. Lett. B 651 (2007) 374-379, arXiv: 0604261 [hep-ph] .

[4] M. J. Strassler and K. M. Zurek, Phys. Lett. B 661 (2008) 263-267, arXiv: 0605193 [hep-ph] . 
[5] ATLAS Collaboration, JINST 3, S08003 (2008).

[6] ATLAS Collaboration, Phys. Lett. B 743 (2015) 15-34, arXiv.org:1501.04020 [hep-ex].

[7] ATLAS Collaboration, JINST 8 (2013) P07015, arXiv.org:1305.2284 [hep-ex] .

[8] M. Cacciari, G. P. Salam and G. Soyez, JHEP 0804 (2008) 0633, arXiv: 0802.1189 [hep-ph] .

[9] A. Read, J. Phys. G 28 (2002) 2693.

[10] ATLAS Collaboration, Phys. Rev. D 92 (2015) 012010, arXiv.org:1504.03634 [hep-ex].

[11] ATLAS Collaboration, ATLAS-CONF-2010-069, 2010 . 